\title{
A Comparison of Methods to Count Breathing Frequency
}

\author{
Atsushi Takayama, Taro Takeshima, Yutaka Nakashima, Takahiro Yoshidomi, \\ Takahiko Nagamine, and Kazuhiko Kotani
}

\begin{abstract}
BACKGROUND: Counting breaths for a full minute for all patients to determine breathing frequency could result in excessive work load for many medical staff. The aim of this study was to verify the agreement of 2 quick screening methods with counting breaths for a full minute. METHODS: We conducted a cross-sectional study to compare the breathing frequency estimates from a 15-s period multiplied by 4 (15-s quadruple) and a value which is 60 divided by the time measured for a single breath (ie, breathing time measurement) against counting breaths for a full minute. Subjects of this study included 58 nurses; 1 nurse acted as the patient, and 57 nurses counted the patient's breathing frequency using each of the 3 methods. Each nurse examiner performed the breathing time measurement, the 15-s quadruple method, and the 1-min breath count, in that order. We performed correlation and Bland-Altman analyses between the 15-s quadruple and 1-min breath count methods, and between the breathing time measurement and 1-min breath count methods. Using paired $t$ tests, we compared the absolute difference between the 15-s quadruple and the 1-min breath count methods to the absolute difference between the breathing time measurement and the 1-min breath count methods. RESULTS: The coefficient of correlation between the 15-s quadruple and 1-min breath count was 0.83 , while the coefficient of correlation between the breathing time measurement and 1-min breath count methods was 0.90 . Brand-Altman analysis indicated that the bias of 15-s quadruple method to the 1-min breath count method was $-2.1 \pm 2.9 \mathrm{SD}$, and the limit of agreement was \pm 5.6 ; the bias of the breathing time measurement method to the 1 -min breath count method was $0.5 \pm 2.6 \mathrm{SD}$, and the limit of agreement was $\pm \mathbf{5 . 0}$. There were statistically significant differences between the 15 -s quadruple and 1-min breath count methods and between the breathing time measurement and 1-min breath count methods $(P<.001)$. CONCLUSIONS: The breathing time measurement method had better agreement with the 1-min breath count method than did the 15-s quadruple method in this study setting. Key words: physical examination; vital signs; breathing frequency; tachypnea; work load; mass screening. [Respir Care 2019;64(5):555-563. () 2019 Daedalus Enterprises]
\end{abstract}

\section{Introduction}

The 4 traditional vital signs, which are pulse rate, body temperature, blood pressure, and breathing frequency, are fundamental factors used to assess the condition of patients worldwide. ${ }^{1}$ Because altered breathing frequency especially tends to be the first sign of clinical deterioration, it is one of the most significant indicators for predicting clinical outcomes. ${ }^{2-6}$ Therefore, breathing frequency has

\footnotetext{
pan. Dr Nakashima is affiliated with the Support Center for Rural Medicine, Yamaguchi Grand Medical Center, Houfu, Yamaguchi, Japan. Dr Yoshidomi is affiliated with the Division of Internal Medicine, Shimonoseki Municipal Toyota Central Hospital, Shimonoseki, Yamaguchi, Japan. Dr Nagamine is affiliated with the Sunlight Brain Research Center, Houfu, Yamaguchi, Japan.
}

The authors have disclosed no conflicts of interest. 
been gaining importance and has been widely incorporated into many different clinical prediction rules. ${ }^{7-9}$

Despite the clinical importance of breathing frequency, many previous studies have pointed out the unreliability of this vital sign. ${ }^{10-12}$ The standard method for assessing breathing frequency is to count for $30 \mathrm{~s}$ or $60 \mathrm{~s} .{ }^{13-15} \mathrm{How}-$ ever, the duration of counting can increase the time needed to perform this assessment in daily clinical situations; hence, many medical staff unofficially use a faster method without any published evidence. ${ }^{16-20}$ Previous studies have assumed that one of the reasons for the unreliability of breathing frequency counts is this unofficial use of various quick-check methods. ${ }^{11,12,21}$ To our knowledge, however, there is no evidence regarding the agreement of these quick-check methods with either of the standard methods. Therefore, it is unclear whether the use of quick-check methods causes the aforementioned unreliability, in the scientific sense of the term.

The aim of this study was to assess the agreement of 2 representative quick-check methods with the accepted standard method of counting breaths for a full minute (Fig. 1). One of the quick-check methods is to count breaths for $15 \mathrm{~s}$ and multiply this count by 4 (ie, 15 -s quadruple), which is unofficially used in many health care facilities. ${ }^{2,11,22}$ The other is quick-check method assessed in this study is the newly developed method in which the provider counts the time needed for a single breath and then divides 60 by the time for a single breath (ie, the breathing time measurement). Our study was the first to provide evidence regarding the validity of these quick-check methods.

\section{Methods}

\section{Study Design}

This cross-sectional study evaluated the agreement of 2 quick-check methods with the 1-min breath count method. First, we calculated the correlation between the 15-s quadruple and 1-min breath count methods, and between the breathing time measurement and 1-min breath count methods. Second, we performed Bland-Altman analyses to compare the 15-s quadruple and 1-min breath count methods, and to compare the breathing time measurement and 1-min breath count methods. Third, we used paired $t$ tests to compare the absolute difference between the 15-s quadruple and 1-min breath count methods, and the difference

Correspondence: Atsushi Takayama MD, Department of Family Medicine, Iwakuni Municipal Miwa Hospital, 1776 Shibukuma Miwa, Iwakuni, 740-1225, Yamaguchi Prefecture, Japan. E-mail: m05054at@live.jp.

DOI: $10.4187 /$ respcare.06451

\section{QUICK LOOK}

\section{Current knowledge}

Assessing breathing frequency using a 30-s count multiplied by 2 tends to yield higher results than those found when counting for 1 full minute. However, many clinicians unofficially use a much quicker method for assessing breathing frequency (counting breaths for $15 \mathrm{~s}$ and then multiplying by 4), without any published evidence to support its validity.

\section{What this paper contributes to our knowledge}

Both the 15-s count multiplied by 4 (15-s method) and the breathing time measurement method had good agreement with 1-min breathing frequency. However, the 15-s method tended to yield higher results than the true value, and the breathing time measurement method had better agreement with 1-min breathing frequency than the 15-s method.

between the breathing time measurement and 1-min breath count methods.

\section{Ethical Approval}

The institutional reviewed board at Jichi Medical University approved this study. The participants provided informed consent.

\section{Setting}

We hosted a training session to train nurses in vital sign assessment at the Toyota Central Hospital on March 26, 2014. In this training session, all subjects practiced the accepted standard of 1-min breath count as well as the 2 quick-check methods. After a 30-min lecture and practicing the breathing frequency methods, we conducted a simple competency test from March 26 to April 28 to assess improvement in the subjects' skills. Subjects counted the breathing frequency of 1 examinee nurse. We collected data on all 3 different breathing frequency methods. This study was conducted to re-assess this data set retrospectively.

\section{Participants}

The 58 subjects ( 57 examiners and 1 examinee) were employed at the Toyota Central Hospital. The mean \pm SD age of the examiners was $40.3 \pm 10.0 \mathrm{y}$, and the examiners' mean \pm SD years of experience was $19.1 \pm 10.2$. Five of the examiners were male ( $8.8 \%$ of all examiners). The 

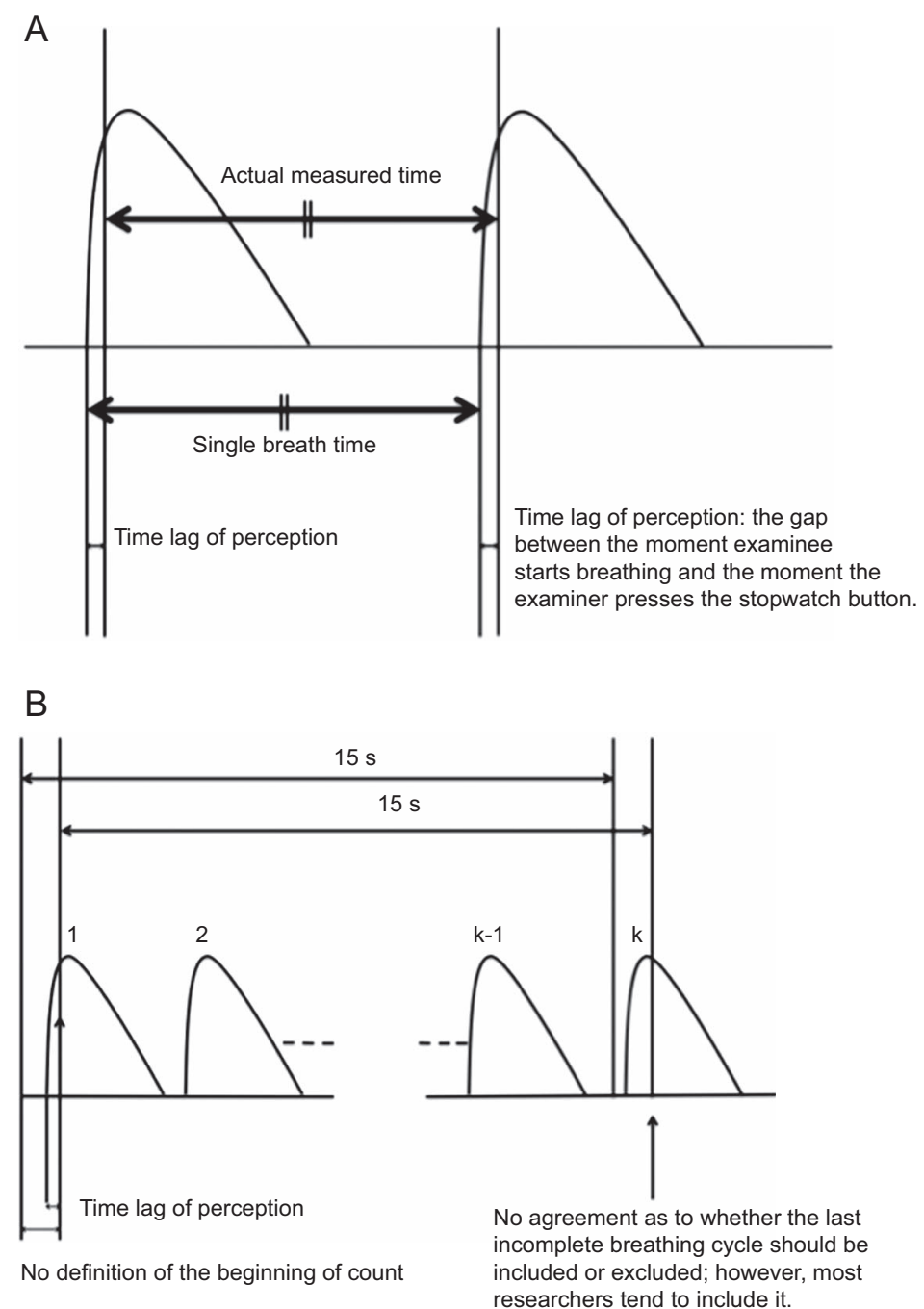

C

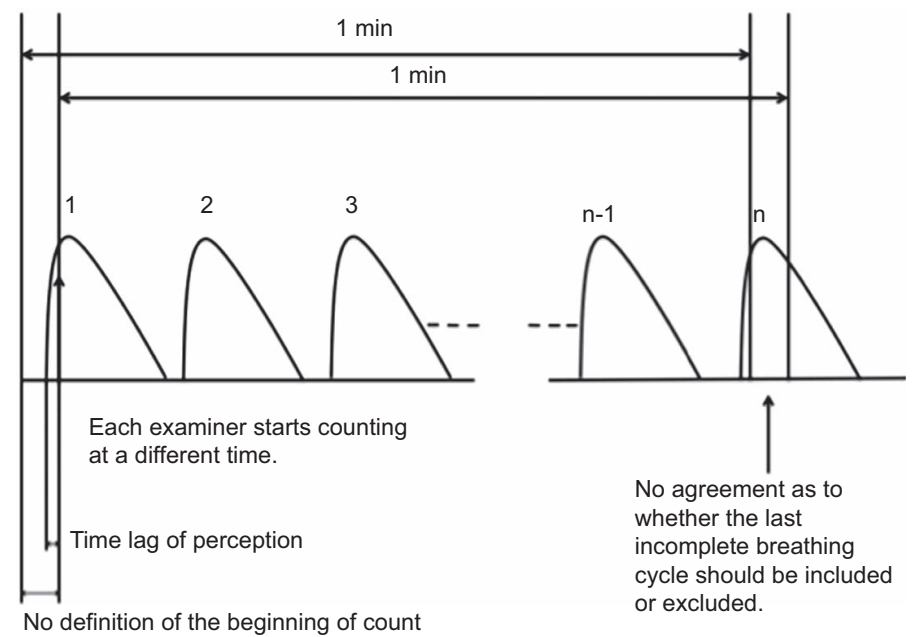

Fig. 1. Schematic representations of the 3 breathing frequency counting methods. A: Breathing time measurement. B: 15-s quadruple. C: 1-min breath count (the accepted standard). 
57 examiners underwent the competency test and were enrolled in this study. We recruited one nurse as an examinee. He was a 48-y-old healthy man, without any particular medical history. He had a smoking habit of 1 pack per day for 28 years, and he volunteered for this role without any particular reason. The other 57 nurses assessed his breathing frequency as examiners.

\section{Study Protocol}

The examinee nurse was instructed to lie down on the bed and close his eyes so that he would not notice when the examination started. He was instructed to change the frequency of his breathing randomly for each session, and to maintain that frequency throughout the session. The breathing frequency in each session was assessed by 1 examiner at a time, and 2-3 examiners underwent the competency test each day. The examiners assessed breathing frequency using each of the 3 methods in a specific order. First, the examiners measured breathing time measurement, followed by the 15 -s quadruple method, and then by the 1-min breath count method. We ensured that the examinee maintained a consistent respiratory pattern during each session using an electronic bedside monitor (PVM2703, Nihon Kohden, Tokyo, Japan). The examiners reported only the duration of 1 breath and the number of breaths in a 15-s period, without calculation. The chronograph used in this study was the TANITA Stopwatch TD392 (Tokyo, Japan), which can measure breathing frequency with an accuracy of one hundredth of a second. We provided brief instructions to the examiners individually before each trial, and we recorded all of the values and supervised each trial. Each nurse participated in only 1 trial, and each method was performed once, unless the process was disturbed by an unavoidable reason, such as the examinee's cough or sneeze, or the examiner not pressing the button.

\section{Measurement of Breathing Frequency}

Breathing Time Measurement. Examiners carefully observed the examinee's breathing pattern, and then recorded inspiratory movement of body parts, generally chest rise movements. Examiners pushed the start button when the cycle of thoracic lifting movements initiated and pushed the stop button when the next cycle of thoracic lifting movements initiated. This means that the duration between the beginning of inspiration and the beginning of the next inspiration, as perceived by the examiner, was measured. We termed this duration the single-breath time. The estimated breathing frequency by the breathing time measurement method is the 60 divided by single-breath time (leading to an accuracy of one hundredth of a second) for the calculation, and rounded off the values to 2 places after the decimal to estimate breathing frequency by the breathing time measurement method.

15-s Quadruple. The breath count during an arbitrarily selected 15-s period was measured. We did not apply any specific criteria for beginning the measurement or for the last incomplete respiratory cycle in this method because there are no previous studies defining such parameters for the 15-s quadruple method. Breathing frequency was estimated by multiplying the 15 -s quadruple breath count by 4 . We treated the estimated breathing frequency from the 15 -s quadruple method as natural numbers.

1-Min Breath Count. We adopted standard measurement criteria for the 1-min breath count, as described in the World Health Organization (WHO) Acute Respiratory Infection program. ${ }^{23}$ Despite our best efforts to obtain the exact definition of the formal measurement of breathing frequency, there were no definitive indications regarding the beginning of the measurement and whether the last incomplete respiratory cycle should be included. Although there is no specific published definition, breathing frequency should implicitly be considered as an integer.

\section{Statistical Analysis}

The mean values of each breathing frequency method were compared using paired Student $t$ tests. We analyzed the correlation between methods using Pearson's product moment correlation, and then we directly compared these coefficients.

We also performed Bland-Altman analyses comparing the various methods to assess breathing frequency. ${ }^{24}$ The Bland-Altman analyses examined the agreement between 2 methods designed to measure breathing frequency. We plotted the differences and bias between the breathing frequency methods, and we calculated upper and lower limits of agreement. The limits of agreement are calculated as \pm $1.96 \mathrm{SD}$ of the mean difference between any 2 methods.

The graphs indicate agreement across the range of breathing frequency values and the yield measures of bias between the breathing frequency estimated with the 15-s quadruple method and the 1-min breath count method, and between the breathing frequency estimated with the breathing time measurement method and the 1-min breath count method. The Bland-Altman spot has 3 abscissa lines. The centerline indicates the mean difference and the upper and lower lines show the limits of agreement. The limits of agreement are calculated as $\pm 1.96 \mathrm{SD}$ of the mean difference between the 2 methods.

We also assessed the absolute value of the differences between various combinations of the methods using Welch's paired $t$ test. Prior to this calculation, we confirmed that both quick-check methods met normality cri- 


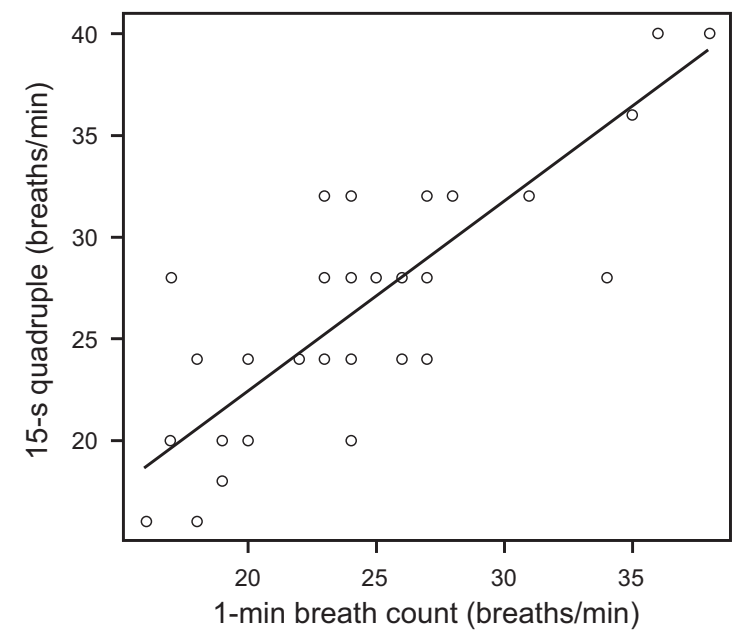

Fig. 2. Scatter plot of the correlation between breathing frequency estimated with the 15-s quadruple method and the 1-min breath count $\left(r=0.83, r^{2}=0.68,95 \% \mathrm{Cl} 0.72-0.89, P<.001\right)$.

teria using the Shapiro-Wilk test. All $P$ values were 2-tailed, with $P<.05$ considered statistically significant.

All statistical analyses were performed using EZR (Saitama Medical Center, Jichi Medical University, Saitama, Japan), which is the graphical user interface for $\mathrm{R}$ software (R Foundation for Statistical Computing, Vienna, Austria). ${ }^{25}$

\section{Results}

\section{Breathing Frequency Measured Using 3 Methods}

The mean \pm SD estimates of the examinee's breathing frequency, based on the breathing time measurement, the 15-s quadruple, and 1-min breath count methods were $24.0 \pm 5.6,26.6 \pm 5.5$, and $24.5 \pm 5.1$ breaths $/ \mathrm{min}$, respectively.

The result of the correlation analysis between breathing frequency estimated with the 15-s quadruple and 1-min breath count methods is shown in Figure 2. The coefficient of the correlation between these methods was 0.83 (95\% CI $0.73-0.90, P<.001)$. The $t$ test showed a significant difference between these methods $(P<.001)$.

Bland-Altman plots of the correlation between breathing frequency estimated with the 15-s quadruple and 1-min breath count methods are shown in Figure 3. The mean \pm SD correlation between these methods is $-2.1 \pm 2.9$. The limits of agreement are \pm 5.6 .

The result of the correlation analysis between breathing frequency estimated with the breathing time measurement and the 1-min breath count methods is shown in Figure 4. The coefficient of the correlation between these methods was 0.90 (95\% CI $0.83-0.94, P<.001)$. The $t$ test did not show a significant difference between these methods $(P=.14)$.

Bland-Altman plots of the correlation between breathing frequency estimated with the breathing time measurement and 1-min breath count methods are shown in Figure 5 . The mean $\pm \mathrm{SD}$ correlation between these methods is $0.5 \pm 2.6$. The limits of agreement are \pm 5.0.

The average correlation between the 15-s quadruple method and the 1-min breath count method is $2.7 \pm 2.2$, while that between the breathing time measurement method and the 1 -min breath count method is $1.9 \pm 1.8$. The paired $t$ test showed a significant difference between the 15-s quadruple and 1-min breath count methods and between the breathing time measurement and 1-min breath count methods $(P<.001)$ (Fig. 6).

\section{Discussion}

We found that both the 15-s quadruple method and the breathing time measurement method correlated significantly with the 1-min breath count method. Brand-Altman analysis, however, revealed that the breathing time measurement method had better agreement with the 1-min breath count method; the 15-s quadruple method consistently overestimated breathing frequency compared with the 1-min breath count method, and its limits of agreement were larger than those for the breathing time measurement method. Further, the difference between the breathing frequency estimated with the breathing time measurement and the 1-min breath count was significantly smaller than the difference between the breathing frequency estimated with the 15-s quadruple method and the 1-min breath count, demonstrating a better agreement between the breathing time measurement method and the 1-min breath count.

We provide new evidence to show that the 15-s quadruple method fundamentally tends to overestimate breathing frequency. In a study of 97 children under the age of $5 \mathrm{y}$, Simoes et $\mathrm{al}^{14}$ reported that the mean difference between a 30-s doubled method and a pneumocardiogram was $1.72 \pm 8.60$, which was smaller than that of the 15 -s quadruple method despite the wide variety of that study's objectives. These results imply that the 15 -s quadruple method leads to excessive counting compared to the 30-s doubled method. A study by Gadomski et al ${ }^{15}$ concluded that the 30-s doubled method tended to yield breathing frequency estimates that were $2-4$ breaths greater than the 1-min breath count, particularly when breathing frequency was high. We believe the 15 -s quadruple method leads to similar overestimates.

We suggest 2 reasons to explain this tendency. First, the 15 -s quadruple estimates are calculated by multiplying the value obtained in $15 \mathrm{~s}$ by 4 , which amplifies any residual error by 4 . Second, there is no consensus for the definition of counting a breath in the 15-s quadruple method. This 


\section{Comparison of Breath-Counting Methods}

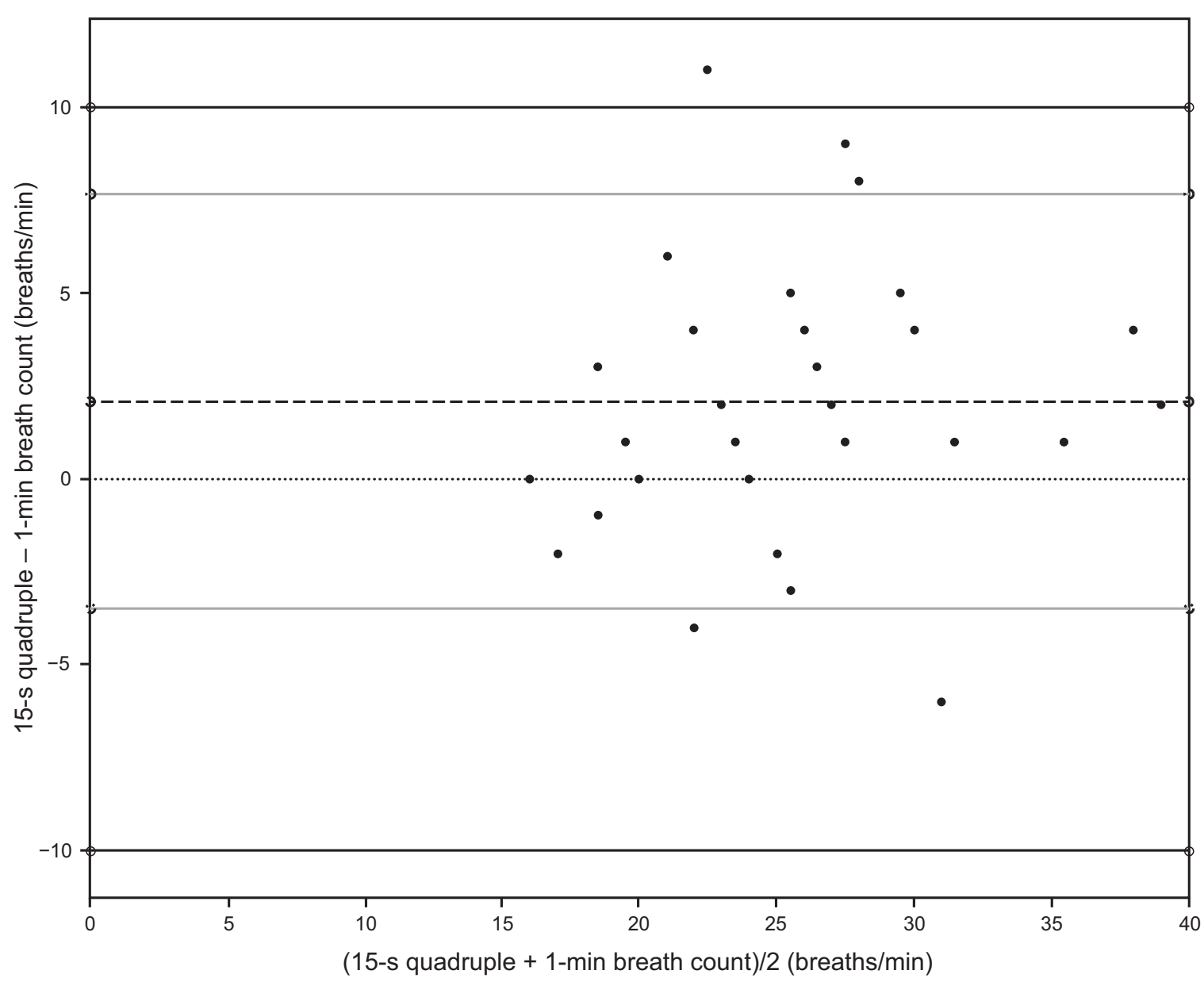

Fig. 3. Bland-Altman spot distribution between the 15-s quadruple method and the 1-min breath count against the mean of measurement. Outer lines are for comparison, upper and lower limits of agreement (gray lines) are equal to the average difference $\pm 1.96 \mathrm{SD}$, and the dashed line indicates the bias.

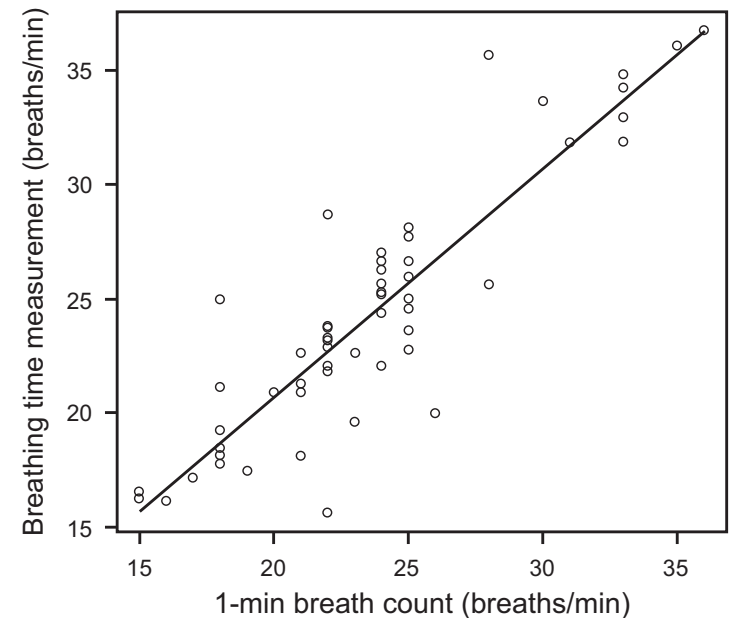

Fig. 4. Scatter plot of the correlation between breathing frequency estimated with the breathing time measurement method and the 1 -min breath count $\left(r=0.90, r^{2}=0.79,95 \% \mathrm{Cl} 0.83-0.93, P<.001\right)$. allows variations such as when an examiner identifies that start of a respiration, and an examiner may count the last incomplete breathing cycle. These variations lead the 15 -s quadruple method to include several additional breathing cycles when the value is multiplied by 4 to represent a full minute of breathing.

One of the most important methodological difficulties for measurement of regular breathing frequency is the need to view the watch or chronometer regularly to track the elapsed time. ${ }^{16}$ Many researchers agree that such methodological multi-tasking difficulties cause inaccuracy. ${ }^{16-22,26-28}$ However, the breathing time measurement method is an extremely quick method and does not require multi-tasking. Although this method is more demanding in terms of calculation, we believe that it can be used as a reliable quick-check method for screening. Complicated calculations are not necessary if the breathing time measurement method is used simply as a screening tool. The singlebreath time is suitable for assessing the patient's breathing frequency quickly during screening. For instance, if the 


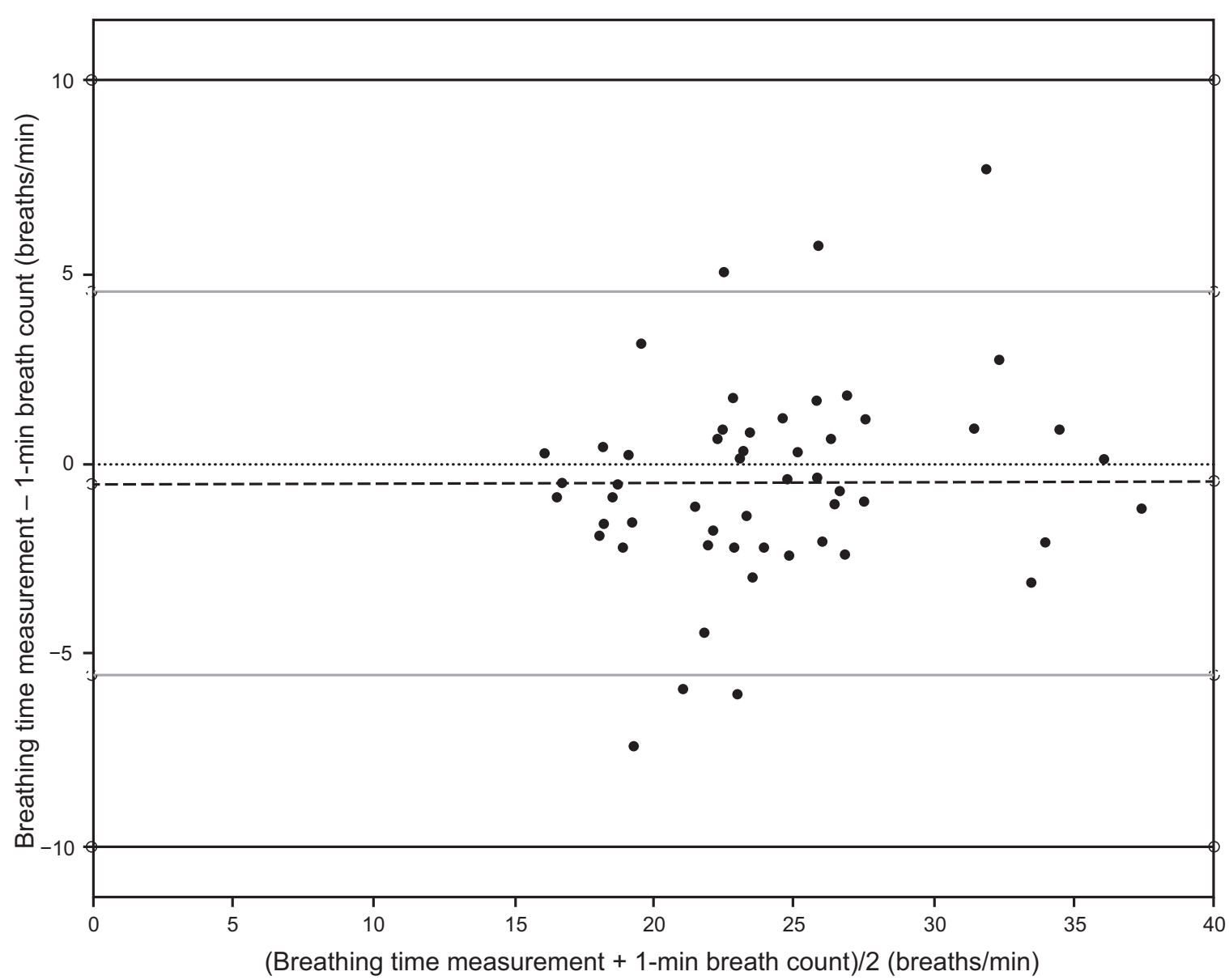

Fig. 5. Bland-Altman spot distribution between the breathing time measurement method and the 1-min breath count. Outer lines are for comparison, upper and lower limits of agreement (gray lines) are equal to the average difference $\pm 1.96 \mathrm{SD}$, and the dashed line indicates the bias.

single-breath time of the patient is $<3 \mathrm{~s}$, there is a high possibility of tachypnea. We used the estimated breathing frequency by breathing time measurement, which requires complex calculations, only for the sake of comparison in this study.

The idea for breathing time measurement, our newly invented spot assessment, is based on the assumption that breathing frequency generally involves regular cycles, initiated by the medullary respiratory center through the Hering-Breuer reflex. ${ }^{29}$ However, as is well known, many types of abnormal respiratory rhythms, such as CheyneStokes, periodic, ataxic, apneustic, Biot's, and gasping respiration, are observed in severely deteriorated cases. ${ }^{30} \mathrm{Be}-$ cause very little is known regarding the variability of the respiratory interval in any given patient, the breathing time measurement method is not always an adequate method to assess abnormal breathing patterns.

Many types of electronic devices have been invented to measure breathing frequency automatically and represent one of the ideal solutions to reduce work load. ${ }^{10}$ Recently, many researchers have started to verify the accuracy of new electronic devices in the clinical setting. The mean \pm SD differences for the breathing time measurement method in our study was comparable to the results obtained with these devices. For instance, the mean \pm SD difference between the readings obtained using respiR8® (Anaxys Technology, Keele, Staffordshire, United Kingdom), Sensium Vitals (Toumaz, Abingdon, United Kingdom), and RespiraSense (PMD Solutions, Cork, Ireland) and the manual method were $0.86 \pm 2.5,0.4 \pm 6.7$, and $-0.41 \pm 1.8$, respectively. ${ }^{31-33}$ The Sensium Vitals system was also studied by other researchers, who found the mean $\pm \mathrm{SD}$ difference to be $0.3 \pm 5.87 .{ }^{34}$ Although these results were obtained in different settings and cannot be compared directly, it seems that our quick-check method is relatively accurate compared with digital patch devices. In addition, it has a low cost and is reasonably quick. At the time of this writing, these devices have not met current clinical standards for accuracy and cost-effectiveness. ${ }^{32}$

Although the 1-min breath count method is widely considered the accepted standard, 3 studies have reported inter-observer differences. One study showed that the 


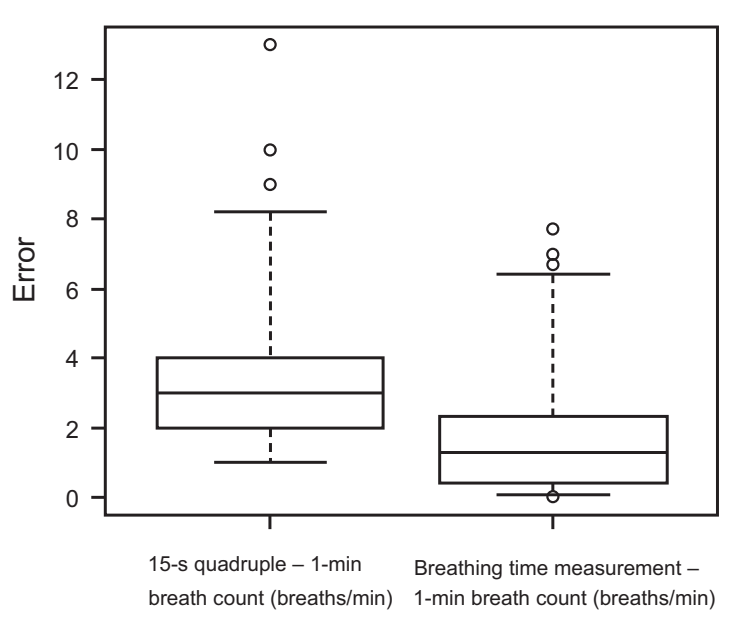

Fig. 6. Comparison of the absolute differences between the breathing time measurement method and the 1-min breath count, and between the 15-s quadruple method and the 1-min breath count. The result of paired $t$ tests between the absolute differences is shown $(P<.001)$. Center lines represent the median. The bottom and top of the boxes are first and third quartiles, respectively. The bottom and top whiskers indicate the 5th and 95th percentiles, respectively. Points show outliers of whisker definition.

mean \pm SD difference for inter-observer agreement among 3 different examiners performing the WHO standard counting was $0.58 \pm 5.4 .{ }^{35}$ This study's observers separately assessed the breathing frequency of 55 subjects, whose ages ranged from $10 \mathrm{~d}$ to $7 \mathrm{y}$, within $30 \mathrm{~min}$. This mean difference is larger than that of the breathing time measurement method in our study, but the authors concluded that this difference was clinically acceptable. The second study showed that the mean \pm SD difference between a standard approach over $60 \mathrm{~s}$ and electronic measurements was $1.7 \pm 1.1 .{ }^{34}$ The third study examined 50 acutely admitted adult subjects in the medical ward. The mean difference derived from widely used electronic measurements was also much larger than that for the breathing time measurement method. This study verified the accuracy of breathing frequency assessment performed by physicians. ${ }^{32}$ The mean \pm SD difference between true breathing frequency assessed by watching a video and the physician's assessment was $-5.4 \pm 10.7$, even after formal training in the manual method, which was a considerably high range of differences. On the basis of these data, it is difficult to conclude that breathing frequency itself is a precise parameter.

Breathing frequency is known worldwide to be an often unreliable vital sign, which suggests a fundamental underlying cause. 17-22,26-28,36 Clinicians should focus more on adequate assessment and use of this important information to enhance patient care. The 1-min breath count method is not completely reliable because breathing frequency itself exhibits a certain range of values depending on the time of day and the patient's condition. In a practical sense, we believe that a screening step before elaborate examination may have many advantages for making clinical decisions, considering feasibility and credibility. Our study is the first step in assessing the agreement of quick-check methods with the accepted standard in methodological terms. Our results show that these methods may be useful for developing quick screening tools to detect tachypnea and bradypnea. Further studies are necessary to assess the feasibility and utility of these in a clinical setting.

\section{Limitations}

This study has limitations. First, our study setting was an experimental scenario meant for training. There was only 1 examinee and this person was healthy, hence this does not represent a patient in distress. Second, the examinee maintained a regular breathing frequency for each test, changing the frequency randomly with each trial. These artificial conditions are different from real-world clinical situations. Third, the examiners did not perform the accompanying calculations while counting breaths for $15 \mathrm{~s}$ and timing the single breath for the breathing time measurement method. This implies that we did not consider human error due to miscalculation and the work burden of calculations. Fourth, the examiners performed 3 different methods sequentially in a very short period of time; although we carefully monitored the breathing patterns of the examinee throughout all the sessions using an electronic bedside monitor, there were minor variations. These limitations should be considered while assessing the clinical suitability of the results. However, the main aim of this study was to assess the methodological agreement of the 2 quick-check methods, thus, we wished to eliminate potential confounders, especially those of disease and human error. These methods should be further investigated in the clinical setting.

\section{Conclusion}

The data from our study suggests that the 15-s quadruple method and the breathing time measurement method were significantly correlated with the 1-min breath count method. However, the 15-s quadruple method fundamentally overestimated breathing frequency. The breathing time measurement method had a better agreement with the 1-min breath count than did the 15-s quadruple method when the participant exhibited a regular breathing frequency.

\section{REFERENCES}

1. Centre for Clinical Practice at NICE (UK). Acutely ill patients in hospital: recognition of and response to acute illness in adults in hospital. London: National Institute for Health and Clinical Excellence (UK), 2007. 


\section{Comparison of Breath-Counting Methods}

2. Gravelyn TR, Weg JG. Respiratory rate as an indicator of acute respiratory dysfunction. JAMA 1980;244:1123-1125.

3. McFadden JP, Price RC, Eastwood HD, Briggs RS. Raised respiratory rate in elderly patients: a valuable physical sign. Br Med J (Clin Res Ed) 1982;284(6316):626-627.

4. Fieselmann JF, Hendryx MS, Helms CM, Wakefield DS. Respiratory rate predicts cardiopulmonary arrest for internal medicine inpatients. J Gen Intern Med 1993;8(7):354-360.

5. Subbe CP, Davies RG, Williams E, Rutherford P, Gemmell L. Effect of introducing the Modified Early Warning score on clinical outcomes, cardio-pulmonary arrests and intensive care utilization in acute medical admissions. Anesthesia 2003;58(8):797-802.

6 . Ridley S. The recognition and early management of critical illness. Ann R Coll Surg Engl 2005;87(5):315-322.

7. Subbe CP, Kruger M, Rutherford P, Gemmel L. Validation of a modified Early Warning Score in medical admissions. QJM 2001; 94(10):521-256

8. Lim WS, Vander Eerden MM, Laing R, Boersma WG, Karalus N, Town GI, et al. Defining community-acquired pneumonia severity on presentation to hospital: an international derivation and validation study Thorax 2003;58(5):377-382.

9. Singer M, Deutschman CS, Seymour CW, Shankar-Hari M, Annane D, Bauer M, et al. The Third International Consensus definitions for sepsis and septic shock (sepsis-3). JAMA 2016;315(8):801-810.

10. Lovett PB, Buchwald JM, Stürmann K, Bijur P. The vexatious vital: neither clinical measurements by nurses nor an electronic monitor provides accurate measurements of respiratory rate in triage. Ann Emerg Med 2005;45(1):68-76.

11. Philip KE, Pack E, Cambiano V, Rollmann H, Weil S, O'Beirne J. The accuracy of respiratory rate assessment by doctors in a London teaching hospital: a cross-sectional study. J Clin Monit Comput 2015; 29(4):455-460.

12. Edmonds ZV, Mower WR, Lovato LM, Lomeli R. The reliability of vital sign measurements. Ann Emerg Med 2002;39(3):233-237.

13. World Health Organization. Fourth Program Report, 1988-1989: ARI Program for control of Acute Respiratory Infections, Geneva, Switzerland: WHO, 1990:31.

14. Simoes EA, Roark R, Berman S, Esler LL, Murphy J. Respiratory rate: measurement of variability over time and accuracy at different counting periods. Arch Dis Child 1991;66(10):1199-1203.

15. Gadomski AM, Khallaf N, el Ansary S, Black RE. Assessment of respiratory rate and chest indrawing in children with ARI by primary care physicians in Egypt. Bull World Health Organ 1993;71(5):523527.

16. World Health Organization. Fourth Program Report, 1988-1989: ARI Program for control of Acute Respiratory Infections, Geneva, Switzerland: WHO, 1990:312.3.1.

17. Hogan J. Why don't nurses monitor the respiratory rates of patients? Br J Nurs 2006;15(9):489-492.

18. Ansell H, Meyer A, Thompson S. Why don't nurses consistently take patient respiratory rates? Br J Nurs 2014;23(8):414-418.

19. Philip K, Richardson R, Cohen M. Staff perceptions of respiratory rate measurement in a general hospital Br J Nurs 2013;22(10):570574.
20. Cardona-Morrell M, Prgomet M, Lake R, Nicholson M, Harrison R, Long $\mathrm{J}$, et al. Vital signs monitoring and nurse-patient interaction: a qualitative observational study of hospital practice. Int J Nurs Stud 2016;56:9-16

21. Hooker EA, O'Brien DJ, Danzl DF, Barefoot JA, Brown JE. Respiratory rates in emergency department patient. J Emerg Med 1989; 7(2):129-132

22. Bianchi W, Dugas AF, Hsieh YH, Saheed M, Hill P, Lindauer C, et al. Revitalizing a vital sign: improving detection of tachypnea at primary triage. Ann Emerg Med 2013;61(1):37-43.

23. World Health Organization. Fourth Program Report, 1988-1989: ARI Program for control of Acute Respiratory Infections, Geneva, Switzerland: WHO, 1990:313.2.1.

24. Bland JM, Altman DG. Statistical methods for assessing agreement between two methods of clinical measurement. Lancet 1986;1(8476): 307-310.

25. Kanda Y. Investigation of the freely available easy-to-use software EZR for medical statistics. Bone Marrow Transplant 2013;48(3): 452-458.

26. Cretikos MA, Bellomo R, Hillman K, Chen J, Finfer S, Flabouris A. Respiratory rate: the neglected vital sign. Med J Aust 2008;188(11): 657-659.

27. Gravel J, Opatrny L, Gouin S. High rate of missing vital signs data at triage in a paediatric emergency department. Paediatr Child Health 2006;11(4):211-2115.

28. Semler MW, Stover DG, Copland AP, Hong G, Johnson MJ, Kriss MS, et al. Flash mob research: a single-day, multicenter, residentdirected study of respiratory rate. Chest 2013;143(6):1740-1744.

29. Hering, KEK. Die Selbststeuerung der Athmung durch den Nervus vagus. Sitzungsberichte der kaiserlichen Akademie der Wissenschaften. Wien: Mathematisch-naturwissenschaftliche Classe, 1868, 57 Band, II. Abtheilung:672-677.

30. Whited L, Graham DD. Abnormal Respirations. Treasure Island, FL: StatPearls Publishing, 2018-2017 Dec 13. Available at https:// www.ncbi.nlm.nih.gov/books/NBK470309/

31. Smith I, Mackay J, Fahrid N, Krucheck D. Respiratory rate measurement: a comparison of methods. Br J Healthcare Assis 2011; $5(1): 18-23$

32. Hernandez-Silveira M, Ahmed K, Ang SS, Zandari F, Mehta T, Weir $\mathrm{R}$, et al. Assessment of the feasibility of an ultra-low power, wireless digital patch for the continuous ambulatory monitoring of vital signs. BMJ Open 2015;5(5):e006606.

33. Lee PJ. Clinical evaluation of a novel respiratory rate monitor J Clin Monit Comput 2016;30(2):175-183.

34. Granholm A, Pedersen NE, Lippert A, Petersen LF, Rasmussen LS. Respiratory rates measured by a standardised clinical approach, ward staff, and a wireless device. Acta Anaesthesiol Scand 2016;60(10): 1444-1452.

35. Lanaspa M, Valim C, Acacio S, Almendinger K, Ahmad R, Wiegand $\mathrm{R}$, Bassat Q. High reliability in respiratory rate assessment in children with respiratory symptomatology in a rural area in Mozambique. J Trop Pediatr 2014;60(2):93-98.

36. Kory RC. Routine measurement of respiratory rate: an expensive tribute to tradition. J Am Med Assoc 1957;165(5):448-450. 